\title{
CAVERNOMAS
}

\section{Conduta no Serviço de Neurocirurgia da Santa Casa de Belo Horizonte}

\author{
Marcello Penholate Faria ${ }^{1}$, Walter J osé Fagundes-Pereyra², \\ Gervásio Teles Cardoso de Carvalho ${ }^{4}$, Atos Alves de Sousa ${ }^{3}$
}

\begin{abstract}
RESUMO - A melhor compreensão da história natural dos cavernomas, o aprimoramento dos métodos diagnósticose das técnicas microcirúrgicastornaram possíveisas seguintes condutas: tratamento conservador, radiocirurgia e ressecção cirúrgica. Apresenta-se revisão de 33 pacientesoperadosno serviço de Neurocirurgia da Santa Casa de Belo Horizonte, no período de 1992 a 2001. Cavernomas corticais e subcorticais que se manifestaram por crises convulsivas de difícil controle (57,5\%) ou principalmente por hemorragia (15,1\%) foram tratados cirurgicamente. Aslesões profundas (gânglios basais, tálamo e tronco encefálico) somaram 27,7\%. Estas, atualmente, só devem ser operadas quando estão próximasà superfície ependimária ou pial. Indicou-se ainda a ressecção de lesões medulares $(5,5 \%)$ e cerebrais profundas, que apresentaram déficit focal progressivo(13,8\%) ou episódios recorrentes de hemorragia(13,8\%). Aslesões pequenase profundas que não apresentam hemorragia devem ser tratadas conservadoramente.Não existe, no momento, evidência de resultados favoráveis com a radiocirurgia.
\end{abstract}

PALAVRAS-CHAVE: cavernomas, quadro dínico, microcirurgia.

\section{Cavernous malformations: surgical management in Belo Horizonte Santa Casa Hospital}

ABSTRACT - The better understanding of the natural history of the cavernous malformations and the improvement of diagnostic methods and of microsurgical techniques have made the management of cavernous malformations possible through the conservative treatment, radiosurgery, and microsurgical resection. We present 33 cases operated at our service at Santa Casa Hospital, Belo Horizonte, from 1992 to 2001 . Cortical and subcortical cavernomasmanifested by epilepsy (57.5\%) or mainly by hemorrhage (15.1\%) were surgically approached. The deep lesions (basal ganglia, talamo and brain steam) represented $27.7 \%$ of our cases. They should only be operated when located near the pial or ependimary surface. The resection of spinal cord lesions ( $5.5 \%$ ) and of deep brain lesions is also recommended when they present progressive focal deficit (13.8\%) or recurrent episodesof hemorrhage (13.8\%). Small and deep seated cavernomasthat do not present bleeding must be conservatively treated. There has been no evident favourable result related to radiosurgery so far.

KEY WORDS: cavernous malformations, clinical presentation, microsurgery.

Asmalformações vasculares do sistema nervoso central (SNC) são anomalias do desenvolvimento não neoplásicas. Podem ser divididas, de acordo com McCormick ${ }^{1}$, em quatro tipos: malformações ateriovenosas (MAVs), telangectasias capilares, malformações venosase hemangiomas cavernosos (HC) ou cavernomas.

Os cavernomas são lesões raras, representam de $5 \%$ a $13 \%$ das malformações vasculares do SNC.
Essa incidência aumentou tendo em vista a maior possibilidade de diagnóstico com o desenvolvimento dos exames de imagem, principalmente a ressonância magnética (RM). Segundo o aspecto à RM, os cavernomas podem ser classificados conforme os critérios de Zabramski². São sobretudo identificados nas sequências ponderadas em $\mathrm{T}^{3}$.

O presente estudo foi realizado com o objetivo de analisar aspectos clínicos, histopatológicos, ra-

Santa Casa de Belo Horizonte - Faculdade de Ciências Médicas de Minas Gerais, Belo Horizonte MG, Brasil: ${ }^{1}$ Neurocirurgião da Santa Casa de Belo Horizonte: ${ }^{2}$ Neurocirurgião e Mestre em Medicina pela Santa Casa de Belo Horizonte; ${ }^{3}$ Neurocirurgião da Santa Casa de Belo Horizonte, Professor da Faculdade de Ciências Médicas de Minas Gerais e Doutor em Neurocirurgia pela Escola Paulista de Medicina; ${ }^{4}$ Neurocirurgião da Santa Casa de Belo Horizonte, Professor da Faculdade de Ciências Médicas de Minas Gerais e Mestre em Neurocirurgia pela Escola Paulista de Medicina.

Recebido 10 Setembro 2003, recebido na forma final 19 Maio 2004. Aceito 12 Julho 2004.

Dr. Marcello Penholate Faria - Rua Penafiel 387 - 30310-420 Belo Horizonte MG - Brasil. E-mail: penholate@hotmail.com 
diológicos e evolutivos de pacientes portadores de HC de diferentes localizações, com revisão da literatura e proposta de protocolo de conduta nos cavernoms.

\section{MÉTODO}

Foram avaliados, retrospectivamente, 33 pacientes portadores de HC. Todos foram tratados no serviço de neurocirurgia da Santa Casa de Belo Horizonte, entre março de 1992 e setembro de 2001. Estudo conduzido com a aprovação da comissão de ética da instituição.

A idade variou de 5 a 70 anos $(M=31,5 \pm X \mu e=y)$. A maior incidência ocorreu na faixa etária de 20 a 40 anos (Tabela 1). Vinte pacientes $(60,6 \%)$ eram do sexo mas culino e $13(39,4 \%)$ do feminino.

Os pacientesforam avaliados quanto a apresentação clínica, presença de hemorragia, características morfológicas da lesão, abordagem cirúrgica, e evolução pósoperatória. O período de seguimento variou de 4 a 120 meses $(M=56,0 \pm X \mu \mathrm{e}=\mathrm{y})$. Utilizaram-se as escalas de resultados de Glasgow para o resultado final no pós-operatório.

Foram utilizados os testes de t de Student e o Quiquadrado, com as correções de Fisher e Yates quando necessário.

\section{RESULTADOS}

A manifestação clínica mais comum foi crise convulsiva $(57,5 \%)$, seguida pelo déficit motor focal $(27,7 \%)$ e pela cefaléia $(18,1 \%)$. Outros sintomas e sinais são apresentados na Tabela 2.

Foram classificados como casos de hemorragia os pacientes que apresentaram um sintoma clínico como cefaléia súbita ou crise convulsiva, associado ou não a déficit ou sinal focal, confirmadosà tomografia computadorizada do crânio (TC) com área hiperdensa indicativa de sangramento aguda, à RM com imagens sugestivas de sangramento agudo e, em 2 casos, à punção lombar sugestiva de hemorragia.

Nos dois casos com lesões medulares, um deles manifestou-se clinicamente com paraparesia acentuada e o outro com dor lombar.

Três pacientes apresentavam a forma familiar da doença. Em dois a lesão era assintomatica e se localizava superficialmente no tronco encefalico. Houve ainda associação de cavernoma familiar com aneurisma intracraniano assintomatico em um caso.

Lesões multiplas ocorreram em três casos sendo dois com historia familiar para a doença.

Todos os pacientes foram submetidosa TC. Já a
Tabela 1. Distribuição por idade em 33 casos de cavernomas.

\begin{tabular}{lc}
\hline Idade & $\mathrm{N}(\%)$ \\
\hline 0 a 20 anos & $9(27,2)$ \\
20 a 40 anos & $16(48,5)$ \\
Maiores de 40 anos & $8(24,2)$ \\
Total & $33(99,9)$ \\
\hline
\end{tabular}

Tabela 2. Quadro clínico de 33 casos de cavernoma.

\begin{tabular}{lc}
\hline Sintomas & N $(\%)$ \\
\hline Crise convulsiva & $19(57,5)$ \\
Déficit motor & $9(27,7)$ \\
Cefaléia & $6(18,1)$ \\
Hemorragia & $5(15,1)$ \\
Síndrome cerebelar & $3(9,0)$ \\
Assintomáticos & $2(6,0)$ \\
Distúrbios visuais & $2(6,0)$ \\
Déficit de nervos cranianos & $2(6,0)$ \\
Vertigem & $1(3,0)$ \\
Coma & $1(3,0)$ \\
Dor lombar & $1(3,0)$ \\
Disartria & $1(3,0)$ \\
Síndrome de hipertensão intracraniana & $1(3,0)$ \\
\hline
\end{tabular}

RM encefálica foi realizada em 21 pacientes $(63,6 \%$ ), enquanto a angiografia com subtração digital em 6 casos $(18,1 \%)$.

As lesões no SNC ocorreram nas regiões supratentorial, infratentorial e medular (Tabela 3).

Todos os pacientes foram submetidos a tratamento cirúrgico. Três pacientes com cavernomas localizados no tronco encefálico foram conduzidos inicialmente de forma conservadora. Dois casos foram operados posteriormente após ressangramento e um paciente por apresentar déficit motor em progressão (Figs 1 e 2). A indicação cirúrgica seguiu a proposta de conduta demonstrada nas Figuras 3 e 4.

Craniotomia convencional foi empregada em 27 pacientes e a craniotomia com localização por estereotaxia em quatro pacientes $(11,1 \%$ ) (Figs 5 , 6 e 7). Os dois pacientes com lesões medulares foram submetidos a laminectomia com ressecção microcirúrgica do cavernoma. 
Tabela 3. Distribuição da localização de 36 cavernomas em 33 pacientes.

\begin{tabular}{lcc}
\hline Localização & N & $(\%)$ \\
\hline Supratentorial & 28 & $(77,7)$ \\
Frontal & 10 & $(27,7)$ \\
Parietal & 7 & $(19,4)$ \\
Temporal & 6 & $(16,6)$ \\
Occipital & 4 & $(11,1)$ \\
Intraventricular & 1 & $(2,7)$ \\
Infratentorial & 6 & $(16,6)$ \\
$\quad$ Ponte & 4 & $(11,1)$ \\
Bulbo & 1 & $(2,7)$ \\
Cerebelo & 1 & $(2,7)$ \\
Intramedular & 2 & $(5,5)$ \\
$\quad$ Toracolombar & 2 & $(5,5)$ \\
Total & 36 & $(99,8)$ \\
\hline
\end{tabular}

A evolução pós-operatória foi avaliada precocemente, em até 15 dias de pós-operatório, e tardiamente em 4 a 120 meses $(M=56)$.

Três pacientes com lesões supratentoriais sem alteração motora pré-operatória desenvolveram déficit motor focal transitório com recuperação completa. Um paciente evoluiu com alteração da acuidade visual persistente. Nos pacientes com lesões infratentoriais ocorreram alterações de nervos cranianos em dois casos $(6,0 \%)$, em um deste permanente. Um paciente apresentou hematoma extradural no pós-operatório tendo sido necessária nova intervenção, e outro evoluiu com alteraçõescerebelares transitórias (Tabela 4). Um paciente teve infecção de ferida cirúrgica e osteomielite evoluindo com sepse e óbito (3,0\%) (Tabela 5).

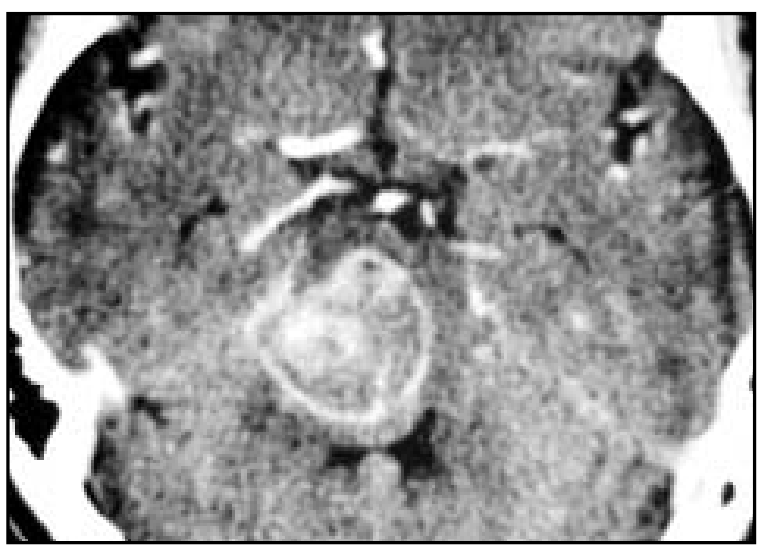

Fig 1. TC de paciente com cavernoma pontino.
Tabela 4. Relação entre complicaçõese localização em 33 casos de cavernomas.

\begin{tabular}{lcc}
\hline Localização & N & $(\%)$ \\
\hline Supratentoriais profundos & 4 & $(12,1)$ \\
$\quad$ Déficit motor & 3 & $(9,0)$ \\
Distúrbios visuais & 1 & $(3,0)$ \\
Supratentoriais superficiais & 0 & \\
Infratentoriais & 5 & $(15,1)$ \\
$\quad$ Déficit de nervos cranianos & 2 & $(6,0)$ \\
Síndrome cerebelar & 1 & $(3,0)$ \\
Hemorragia & 1 & $(3,0)$ \\
$\quad$ Infecção cirúrgica, sepse, óbito & 1 & $(3,0)$ \\
Medulares & 2 & $(6,0)$ \\
$\quad$ Paraparesia & 1 & $(3,0)$ \\
Distúrbios esfincterianos & 1 & $(3,0)$ \\
Total & 11 & $(33,3)$ \\
\hline
\end{tabular}

Tabela 5. Complicações e GOS (Glasgow outcome scale) inicial e tardio em 11 pacientes com cavernomas.

\begin{tabular}{clcc}
\hline Paciente & Complicação & $\begin{array}{c}\text { G.O.S. } \\
\text { inicial }\end{array}$ & $\begin{array}{c}\text { G.O.S. } \\
\text { final }\end{array}$ \\
\hline 1 & Déficit motor & 3 & 4 \\
2 & Déficit motor & 3 & 5 \\
3 & Déficit motor & 3 & 5 \\
4 & Déficit de IX, X,XII & 3 & 2 \\
5 & Infeção, sepsis, óbito & 3 & 1 \\
6 & Déficit de VI,VII & 3 & 3 \\
7 & Distúrbios visuais & 3 & 3 \\
8 & Síndrome cerebelar & 4 & 5 \\
9 & Hemorragia & 3 & 3 \\
10 & Paraparesia & 3 & 3 \\
11 & Distúrbios esfincterianos & 3 & 3 \\
\hline
\end{tabular}

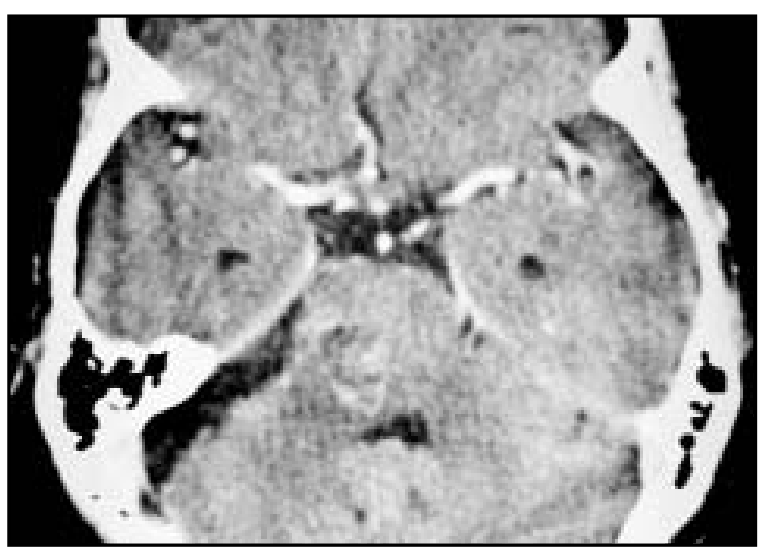

Fig 2. TC de controle pós operatório. 


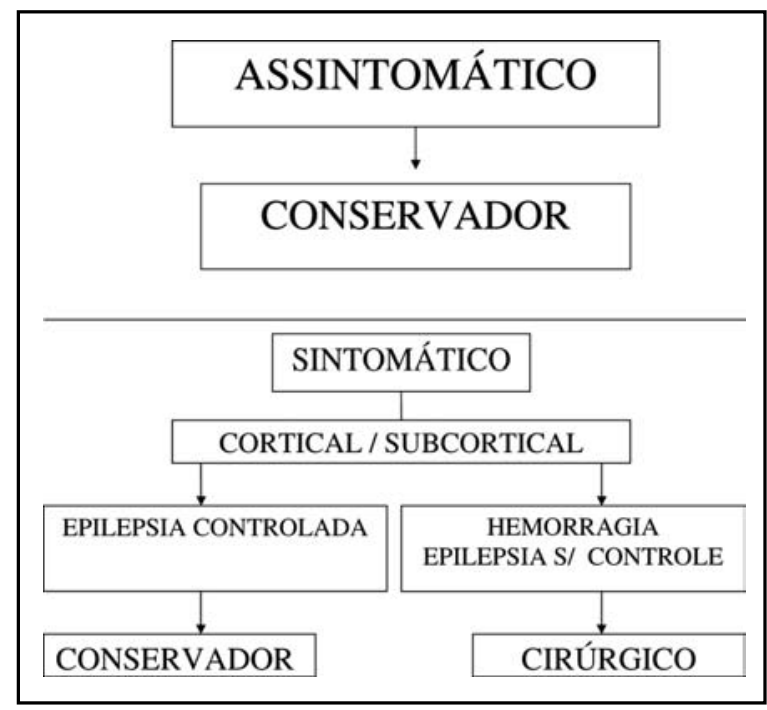

Fig 3. Proposta de conduta geral nos cavernomas.

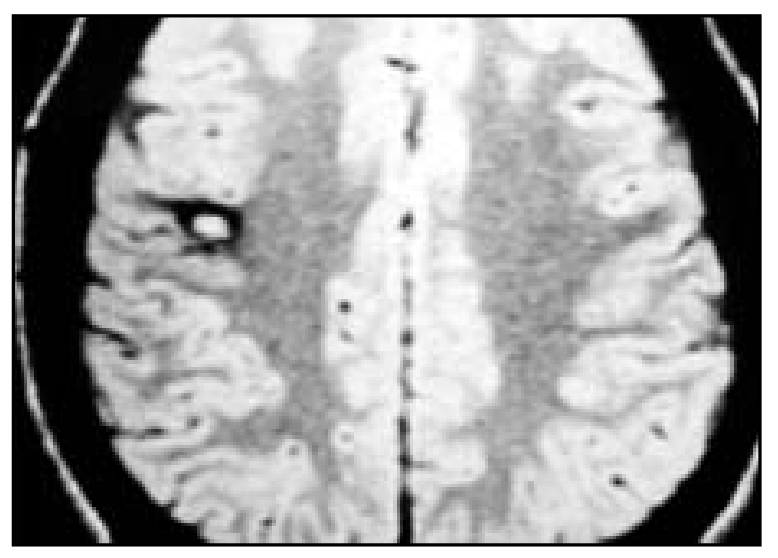

Fig 5. RM de paciente com cavernoma cortical.

\section{DISCUSSÃO}

Osangiomas cavernosos são também conhecidos como hemangiomas cavernosos, malformaçõescavernosasou simplesmente cavernomas. São lesões circunscritas, variam de tamanho, de milímetrosa centímetros ${ }^{4}$ existindo correlação entre seu tamanho e a sintomatologia. Em diversas séries as lesões tornaram-se mais sintomáticas à medida que aumentaram de volume; entretanto; as menores são muito mais freqüentes ${ }^{4}$.

Ascaracterísticas macroscópicastípicas são lesões lobuladas, avermelhadas e arroxeadas, com aspecto de framboesa 5 .O tecido circundante é geralmente descolorado e espesso em decorrência de hemorragias antigas. Apresenta limites bem definidos apesar da ausência de cápsula. À microscopia, comprova-se a ausência de cápsula pelo achado de

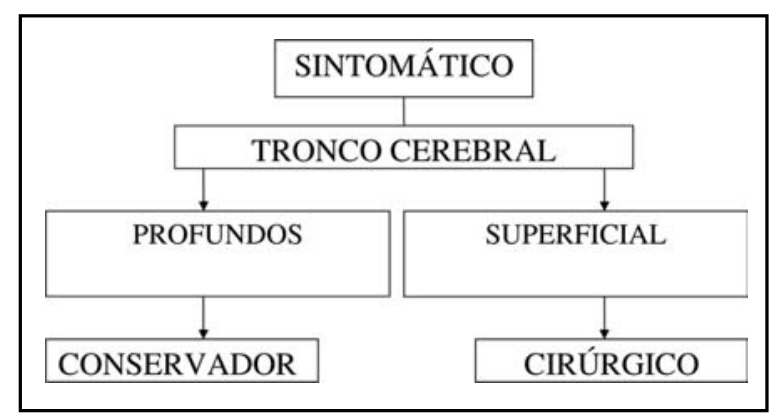

Fig 4. Proposta de conduta geral nos cavernomas.

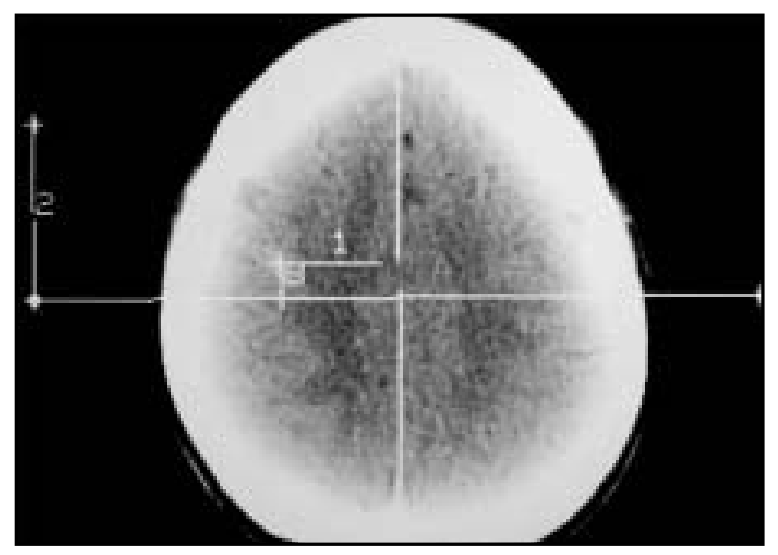

Fig 6. Localização estereotáxica do cavernoma.

áreas de interdigitação em tecido gliótico circundante. Demonstram-se áreas císticas separadas por finos septos. Estes são constituídos de uma camada de endotélio em matriz de colágeno com espaços vasculares dilatados de forma e tamanho variado. Algumas vezes apresentam-se áreas com calcificaçõese areas com depósito de hemossiderina resultado de sangramentosantigos ${ }^{6}$. São malformações crípticas, parcialmente trombosadas ${ }^{7}$. Essas lesões podem ser evidenciadas à TC e de maneira maisprecisa pela RM, mas não são identificadas à angiografia, motivo pelo qual são denominadas "angiograficamente ocultas".

Os cavernomas são lesões raras. Em séries de autópsia corresponderam de $0,02 \% \%^{5,8}$ a $4 \%{ }^{9}$ dasmalformações vasculares do SNC. Já em estudos clínicos existe grande diferença de dados ao longo dos anos pois a incidência aumentou com a melhoria dos métodos de imagem, principalmente através da RM ${ }^{6,8}$. Em revisão da literatura Voigt e Yasargil ${ }^{9}$ não encontraram prevalência de cavernomas em relação a sexo. No presente estudo houve pequena prevalência do sexo masculino (1,66/1) como em outras séries ${ }^{10-12 .}$ 


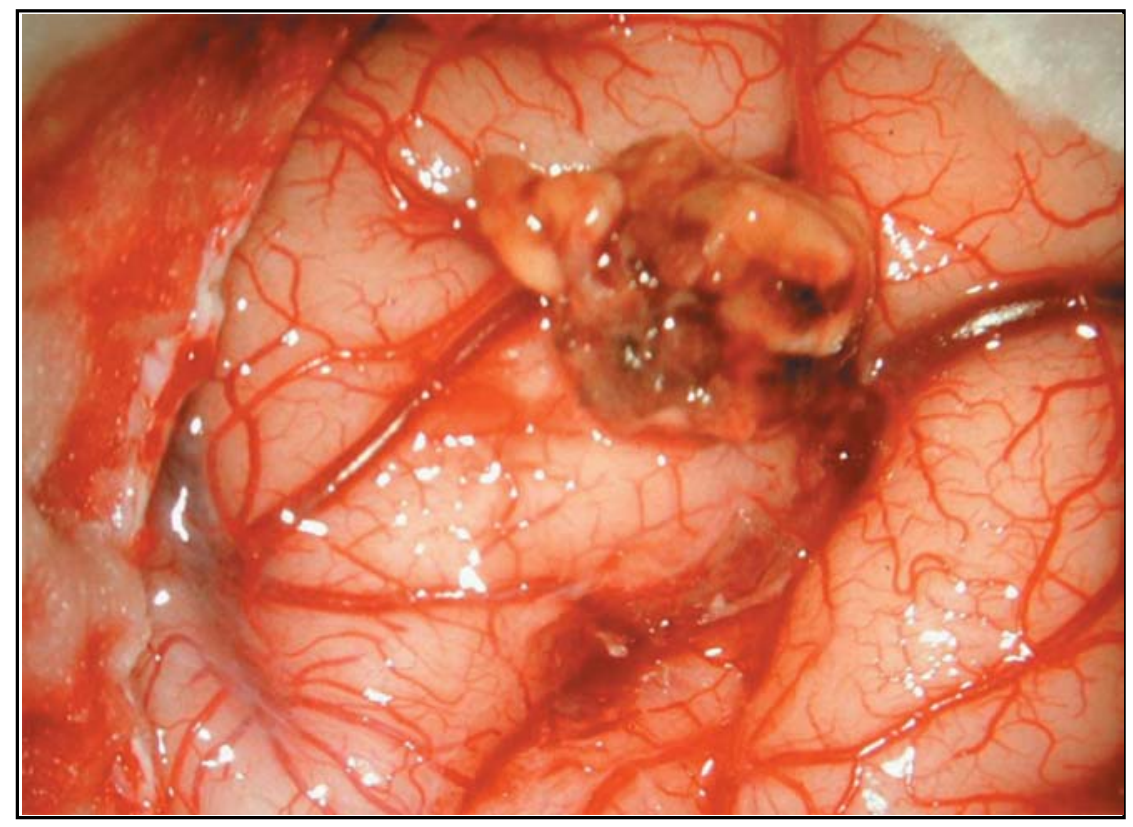

Fig 7. Cavernoma cortical: aspecto pré-operatório.

A maior incidência ocorre nos pacientes entre 20 e 40 anos $^{6}$ (Tabela 2). Entretanto, há descrições de que os cavernomas seriam usualmente diagnosticados entre os 20 e 50 anos com predomínio na quarta década9,11,13.

Classicamente manifestam-se com crises convulsivas, mas a sintomatologia pode ser bastante variável ${ }^{14}$, dependendo da localização e do volume de sangramento. As lesões de localização supratentorial apresentam principalmente crises convulsi$\operatorname{vas}^{11,15,16}$. Os sinais focais são mais freqüentes em lesões infratentoriais. Estas apresentam-se em faixa etária pouco mais avançada ${ }^{16}$.

Em nosso estudo houve grande número de pacientes com déficits motores instalados no íctus. Mas, nas lesões sintomáticas supratentoriaisa convulsão prevaleceu.

A forma familiar da doença foi confirmada em estudo em $1982^{17}$. O número de casos diagnosticados tende a aumentar com o uso cada vez mais difundido de RM para avaliação das formas familiarese às vezes assintomáticas ${ }^{18}$. A incidência familiar chega a $6 \%^{13}$. Acredita-se que a presença de lesões múltiplasem um único indivíduo varia entre as formas familiar e esporádica, sendo mais raras nesta última ${ }^{4,19}$. A associação dasformasmúltiplas em indivíduos de uma mesma família sugere hereditariedade autossômica dominante com penetrância variável ${ }^{13,20}$. Demonstraram-se alterações cromossômicas nas regiões 7q, 7p e 3 q no gen KRT, principalmente em hispânicos e franceses ${ }^{21}$.

Como se percebe nesta série, a distribuição dos cavernomas pelo SNC segue aproximadamente a proporção da massa de tecido nervoso em cada compartimento: $80 \%$ supratentorial, $15 \%$ infratentorial e $5 \%$ medular (Tabela 3).

A localização dos cavernomas tem grande significado clínico e terapêutico. Os cavernomas intraventriculares não apresentam uma imagem típica do halo perilesional hipointenso, o que difuculta o diagnostico pré-operatório ${ }^{3}$.

Indica-se a "ressecção radical e completa quando possível" ${ }^{16}$. As indicações cirúrgicas para os cavernomas são: epilepsia intratável clinicamente, necessidade de diagnóstico, hemorragia recorrente documentada e piora neurológica progressiva. A principal contra-indicação relativa é a localização em áreas eloqüentes como área motora, gânglios basais e tronco cerebral ${ }^{22,23}$. Por se acreditar na necessidade do diagnóstico e na baixa morbidade cirúrgica ${ }^{24}$, houve grande ímpeto para a abordagem maisagressiva destaslesões. Atualmente essa conduta vem sendo reavaliada diante da evolução relativamente benigna demonstrada em estudos recentes ${ }^{25}$.

Nossa indicação cirúrgica seguiu os critérios acima citados conforme protocolo de conduta (Figs 3 e 4). O resultado cirúrgico varia principalmente 
conforme a localização da lesão e quadro clínico do paciente. Aslesões supratentoriaisque se apresentam como epilepsia refratária possuem bom prognóstico. Há séries que demonstram bom resultado em $80 \%$ dos casos operados, com controle a médio e longo prazo ${ }^{24}$. Há de se ressaltar a possibilidade de se definir por eletrocorticografia peroperatória áreas epileptógenas perilesionais para a ressecção adequada ${ }^{24}$ Recomenda-se a excisão da camada gliótica perilesional para melhor resultado clínico ${ }^{26}$. Quanto aos angiomas venosos que geralmente acompanham os $\mathrm{HC}$ considera se importante a sua identificaçao precoce e a sua preservação ${ }^{23,27}$.

Em casos de lesões corticais cuja apresentação clínica é de hemorragia o tratamento consiste na retirada microcirúrgica. Naslesões profundas devese ponderar a indicação microcirúrgica considerando sua localização em relação à proximidade com a superfície pial ou ependimária. Para as lesões localizadasno tronco encefálico e gânglios da base o tratamento deve ser criteriosamente avaliado, opta-se geralmente pela conduta conservadora com acompanhamento neurorradiológico, e em caso de ressangramento ou piora do quadro clínico, indicase a cirurgia. A microcirurgia para estas lesõesé indicada quando, pelo menos em parte, estiverem próximas à superfície pial ou ependimária. Nestes casos preserva-se a área gliótica perilesional ${ }^{28}$.

A indicação da radiocirurgia tem sido questionada inclusive em casos de indicação microcirúrgica limitada pois seus resultados demonstram o controle dos eventos hemorrágicos depois dos dois primeirosanos de seguimento 29,30 . Ao mesmo tempo, estudos da história natural do sangramento dos cavernomas demonstram que as hemorragias ocorrem em salvase que o risco de ressangramento diminui após 2 a 3 anos de tratamento conservador ${ }^{31}$.

Em conclusão, os cavernomas são lesões benignas, cuja história natural ainda não está completamente definida. A cirurgia está indicada visando o controle ou diminuição da freqüência das crises convulsivas, a prevenção de ressangramento e a diminuição dos sintomas causados pelo efeito de massa. A radiocirurgia apresenta ainda resultados desencorajadores. O prognóstico é influenciado principalmente pela localização da lesão.

\section{REFERÊNCIAS}

1. McCormick WF. Classification, pathology and natural history of angiomas of the central nervous system. Neurol Neurosurg, Weekly update: $1975 ; 1$.
2. Zabramski JM, Washer TM, Spetzler RF, et al. The natural history of familial cavernous malformations: results of a ongoing study. J Neurosurg 1994;80:422-432

3. Tatsui CE, Koerbel A, Prevedello DM, et al. Ressonância magenética dos cavernomas intraventriculres Arq Neuropsiquiatr 2003;61:79-82.

4. Robinson JR Jr., Awad IA, Little JR. Natural history of cavernous angioma. J Neurosurg 1991;75:709-714

5. Otten P, Pizzolato GP, Rilliet B, et al. A propos de 131 cas d'angiomes caverneux (cavernomes) du S.N.C., repérés par l'analyse retrospective de 24535 autopsies. Neurochirurgie 1989;35:82-83.

6. Rigamonti D, Spetzler RF. The association of venous and cavernous malformations: report of four cases and discussion of the physiopathologic, diagnostic, and therapeutic implications. Acta Neurochir (Wien) 1988;92:100-105.

7. Shuey HM Jr, Day AL, Quisling RG, et al. Angiografically cryptic cerebrovascular malformations. Neurosurgery 1979;5:476-479.

8. Berry RG, Alpers BJ, White JC. The site structure and frequency of intracranial aneurysms, angiomas, and arteriousvenuos abnormalities. In Millikan CH (ed). Research publications. Association for Research in Nervous and Mental Disease. Baltimore: Willians and Wilkins 1966; 41:4-72.

9. Voigt K, Yasargil MG. Cerebral cavernous hemangiomas or cavernomas: incidence, pathology localization, diagnosis, clinical features and treatment. Review of literature and report of an unusual case. Neurochirurgia (Stuttg) 1976;19:59-68.

10. Kupersmith MJ, Jafar J, Kalish H, et al. Natural history of brainsteam cavernous malformations. Neurosurgery 2001;48:47-54.

11. Yamasaki T, Handa H, Yamshita J, et al. Intracranial and orbital caverous angiomas: a rewiew of 30 cases. J Neurosurg 1986;64:197-208.

12. Giombinni S, Morello G. Cavernous angioma of the brain account of fourteen personal cases and rewiew of literature. Acta Neurochirirgica 1978;40:61-82.

13. Rigamoti D, Hadley MN, Drayer BP, et al. Cerebral cavernous malformation: incidence and familial ocurrence. N Engl J Med 1998;319:343-347.

14. Azevedo MFA, Souza LAM, Leite AC, Rodrigues FF. Distonia segmenta como manifestação de angioma cavernoso. Arq Neuropsiquiatr 2001;59: 613-615.

15. Lynch JC, Andrade R, Pereira C, et al. Angioma cavernoso intracraniano. Arq Neuropsiquiatr 1994;52:237-242.

16. Huhn SL, Rigamonti D, Hsu F. Indications for surgical intervention. In Awad IA, Barow DC (eds). Cavernous malformations. Eds AANS Publications Committee, 1993;87-99.

17. Hayman LA, Evans RA, Ferrell RE, et al. Familial cavernous angiomas: natural history and genetic study over a 5-year period. Am J Med Genet 1982;11:147-1609.

18. Fobe JL, Lima JBN, Buone ML, Correa Neto J. Angioma cavernoso familiar Arq Neuropsiquiatr 1996;54:655-660.

19. Morais JW, Carvalho GTC, Vega MG, Souza AA. Angioma cavernoso associado a aneurisma cerebral: relato de caso. Arq Neuropsiquiatr 1995;14:148-151.

20. Houbert P, Choux M, Houtteville JP. Cerebral cavernomas in children Neurochirurgie 1989;35:104-105.

21. Zhang J, Clatterbuck RE, Rigamonti D, et al. Mutations in KRIT1 in familial cerebral cavernous malformations. Neurosurgery 2000;46:1272-1279.

22. Shah M, Heros RC Microsurgical treatment of supratentorial lesions. In Awad IA, Barow DC (eds). Cavernuos malformations. Eds AANS Publications Committee. 1993;111-116.

23. Wascher TM, Spetzler RF. Microsurgical treatment of infratentorial CM. In Awad IA, Barow DC (eds): Cavernous malformations, Eds AANS Publications Committee. 1993:117-132.

24. Vaquero L, Salazar J, Martinez R, et al. Cavernomas of the central nervous system, clinical syndromes, CT-scan diagnosis, and prognosis after surgical treatment in 25 cases. Acta Neurocchirurg 1987;85:29-33.

25. Curling OD Jr, Kelly DL Jr, Elster AD, et al. An analysis of the natural hystory of cavernous angiomas. J Neurosurg 1991;75:702-708.

26. Steinberg GK, Chang SD, Gewirtz RJ, Lopez JR. microsurgical resection of brainstem, thalamic, and basal ganglia angiographically occult vascular malformations. Neurosurgery 2000;46:260-271.

27. Sarwar M, Mc Cormick WF. Intracerebral venous angioma: case report and review. Arch Neurol 1978;35:323-325.

28. Zimmerman RS, Spetzler RF, Lee KS, Zabramski JM, Hargeaves RW Cavernous malformations of the brain stem. J Neurosurg 1991;75:32-39.

29. Toshinori H, McInerey J, Kondizolka D, Lee JYK, Flickinger JC, Lunsford D. Long- term results after stereotactic radiosurgery for patients with cavernous malformations. Neurosurgery 2002;50:1190-1199.

30. Pollock BE, Garces YI, Stafford SL, Foote RL, Schomberg PJ, Link MJ. Stereotactic radiosurgery for cavernous malformations J Neurosurg 2000:93:987-991.

31. Baker FG, Hanjani AS, Buttler WE, et al. Temporal clustering of hemorrages from untreated cavernous malformations of the central nervous system. Neurosurgery 2001;49:15-32. 